\title{
DETERMINING THE ERRORS IN OUTPUT KINEMATIC PARAMETERS OF PLANAR MECHANISMS WITH A COMPLEX STRUCTURE
}

\author{
W. TRZASKA \\ Cracow University of Technology \\ Faculty of Mechanical Engineering \\ Al. Jana Pawła II 37 \\ 31-864 Kraków, POLAND \\ E-mail: wtrzaska@mech.pk.edu.pl
}

\begin{abstract}
The study is focused on determining the errors in output kinematic parameters (position, velocity, acceleration, jerk) of entire links or their selected points in complex planar mechanisms. The number of DOFs of the kinematic system is assumed to be equal to the number of drives and the rigid links are assumed to be connected by ideal, clearance-free geometric constraints. Input data include basic parameters of the mechanism with the involved errors as well as kinematic parameters of driving links and the involved errors. Output errors in kinematic parameters are determined basing on the linear theory of errors.
\end{abstract}

Key words: errors in kinematic parameters, linear theory of errors, kinematic software library.

\section{Introduction}

Determining kinematic errors in complex mechanisms is a most difficult task. The sources of errors in kinematic parameters include manufacturing errors (when handling linear or angular dimensions), shape and positioning errors, clearances in kinematic pairs, deformations under the force or thermal loads (volumetric or contact strains) and errors associated with drive control. Engineering design of kinematic systems becomes a mutli-stage iterative process, starting from simple (sometimes static) models with simplifying assumptions, moving to more complex ones and finally reaching the model well approximating the behaviour of a real object. While expanding the model, we more accurately define the properties of the investigated system. Nowadays most investigations of complex kinematic models involve the virtual model testing using the FEM approach, MBS (Multibody Simulations) and other simulation programs. The scope of testing is usually defined by the requirements imposed upon the final product and its kinematic systems.

Literature on the subject abounds in works on high-precision kinematic systems, such as manipulators used in surgery (Mavroidis et al., 1998), however most researchers tend to focus on positioning errors (Fenton et al., 1989). This study investigates the position, velocity, acceleration and jerk errors in planar kinematic systems assumed to have rigid links connected by ideal, clearance-free geometric constraints. The investigated system can have any number of drives, but its degree of mobility should be equal to the number of drives. Two drive options are possible: the internal drive or the drive located next to the fixed link. It is further assumed that time in the equations of constraints should not appear explicitly. This assumption does not impose the limit on generality of the analysis because errors due to time variance can be taken into account when analysing errors in kinematic parameters of the driving links. The system has no passive parameters of kinematic constraints or apparent degrees of mobility. Kinematic systems can comprise any number of kinematic units of any class. Bearing in mind that undesired singular positions should not occur in well- designed mechanisms, a further assumption was made that particular error types are 
not interrelated. For brevity of the paper, the example considered in the study is a class II kinematic unit (according to the structural classification by Artobolevsky).

\section{Determining the errors in output kinematic parameters- general case}

\subsection{Position errors}

For a kinematic system whose motion is defined, the equations of position constraints can be given as

$$
\left\{\begin{array}{l}
f_{1}=f_{1}\left(w_{i=1 . . m}, x_{j=1 . . n}, y_{k=1 . . p}\right)=0, \\
f_{2}=f_{2}\left(w_{i=1 . . m}, x_{j=1 . . n}, y_{k=1 . . p}\right)=0, \\
\ldots \\
f_{p}=f_{p}\left(w_{i=1 . . m}, x_{j=1 . . n}, y_{k=1 . . p}\right)=0 .
\end{array}\right.
$$

where

$w_{i=1 . . m}$ - basic (linear or angular) dimensions of the kinematic system known beforehand, these dimensions are independent of time and are included in the set of input data,

$x_{j=1 . . n}$ - time-dependent geometric dimensions of the kinematic system (drives), included in the set of input data and referred to as independent variables

$y_{k=1 . . p}$ - time-dependent link positions (referred to as dependent variables or output quantities), mostly unknown,

The number of equations in the system (2.1) needs to be equal to the number of output quantities. Using the notation

$$
\begin{array}{llll}
\boldsymbol{W} & =\left[\begin{array}{llll}
w_{1} & w_{2} & \ldots & w_{m}
\end{array}\right]^{T}, & \boldsymbol{X}=\left[\begin{array}{llll}
x_{1} & x_{2} & \ldots & x_{n}
\end{array}\right]^{T}, \\
\boldsymbol{Y}=\left[\begin{array}{llll}
y_{1} & y_{2} & \ldots & y_{p}
\end{array}\right]^{T}, & \boldsymbol{F}=\left[\begin{array}{llll}
f_{1} & f_{2} & \ldots & f_{p}
\end{array}\right]^{T} .
\end{array}
$$

the system of Eqs (2.1) can be rewritten in an abbreviated form (Gronowicz, 2003)

$$
F(\boldsymbol{W}, \boldsymbol{X}, \boldsymbol{Y})=\mathbf{0}
$$

In the case of a class II kinematic unit, the nonlinear system of Eqs (2.1) can be solved explicitly. For higher class mechanisms, the system of Eqs (2.1) can be solved by other methods: the Newton-Raphson numerical method or the modification method (Kotulski and Szczepiński, 2004). Solving nonlinear equations is beyond the scope of the present paper, though one has to bear in mind that the choice of the calculation procedures and of the positioning configuration may impact on the adequacy of the solution. Recalling the linear theory of errors, the positioning error equations can be written as 


$$
\left\{\begin{array}{l}
\sum_{i=1}^{m} \frac{\partial f_{1}}{\partial w_{i}} \Delta w_{i}+\sum_{j=1}^{n} \frac{\partial f_{1}}{\partial x_{j}} \Delta x_{j}+\sum_{k=1}^{p} \frac{\partial f_{1}}{\partial y_{k}} y_{k}=0 \\
\sum_{i=1}^{m} \frac{\partial f_{2}}{\partial w_{i}} \Delta w_{i}+\sum_{j=1}^{n} \frac{\partial f_{2}}{\partial x_{j}} \Delta x_{j}+\sum_{k=1}^{p} \frac{\partial f_{2}}{\partial y_{k}} y_{k}=0 \\
\ldots \\
\sum_{i=1}^{m} \frac{\partial f_{p}}{\partial w_{i}} \Delta w_{i}+\sum_{j=1}^{n} \frac{\partial f_{p}}{\partial x_{j}} \Delta x_{j}+\sum_{k=1}^{p} \frac{\partial f_{p}}{\partial y_{k}} y_{k}=0
\end{array}\right.
$$

where: $\boldsymbol{A}_{p 0}, \boldsymbol{B}_{p 0}, \boldsymbol{C}_{p 0}$ - Jacobian matrixes (in further sections these matrixes will not be written in the full form as already defined), $\boldsymbol{\Delta} \boldsymbol{W}$ - column matrix of errors in dimensions $\boldsymbol{W}, \boldsymbol{\Delta} \boldsymbol{X}$ - column matrix of errors in dimensions $\boldsymbol{X}$ and $\boldsymbol{\Delta} \boldsymbol{Y}$ - column matrix of errors in output quantities $\boldsymbol{Y}$.

$$
\begin{gathered}
\boldsymbol{A}_{\boldsymbol{p} \boldsymbol{0}}=\left[\begin{array}{cccc}
\frac{\partial f_{1}}{\partial w_{1}} & \frac{\partial f_{1}}{\partial w_{2}} & \ldots & \frac{\partial f_{1}}{\partial w_{m}} \\
\frac{\partial f_{2}}{\partial w_{1}} & \frac{\partial f_{2}}{\partial w_{2}} & \ldots & \frac{\partial f_{2}}{\partial w_{m}} \\
\ldots & \ldots & \ldots & \ldots \\
\frac{\partial f_{p}}{\partial w_{1}} & \frac{\partial f_{p}}{\partial w_{2}} & \ldots & \frac{\partial f_{p}}{\partial w_{m}}
\end{array}\right], \quad \boldsymbol{B}_{\boldsymbol{p} \boldsymbol{0}}=\left[\begin{array}{cccc}
\frac{\partial f_{1}}{\partial x_{1}} & \frac{\partial f_{1}}{\partial x_{2}} & \ldots & \frac{\partial f_{1}}{\partial x_{n}} \\
\frac{\partial f_{2}}{\partial x_{1}} & \frac{\partial f_{2}}{\partial x_{2}} & \ldots & \frac{\partial f_{2}}{\partial x_{n}} \\
\ldots & \ldots & \ldots & \ldots \\
\frac{\partial f_{p}}{\partial x_{1}} & \frac{\partial f_{p}}{\partial x_{2}} & \ldots & \frac{\partial f_{p}}{\partial x_{n}}
\end{array}\right], \quad \boldsymbol{C}_{\boldsymbol{p} \boldsymbol{0}}=\left[\begin{array}{cccc}
\frac{\partial f_{1}}{\partial y_{1}} & \frac{\partial f_{1}}{\partial y_{2}} & \ldots & \frac{\partial f_{1}}{\partial y_{p}} \\
\frac{\partial f_{2}}{\partial y_{1}} & \frac{\partial f_{2}}{\partial y_{2}} & \ldots & \frac{\partial f_{2}}{\partial y_{p}} \\
\ldots & \ldots & \ldots & \ldots \\
\frac{\partial f_{p}}{\partial y_{1}} & \frac{\partial f_{p}}{\partial y_{2}} & \ldots & \frac{\partial f_{p}}{\partial y_{p}}
\end{array}\right], \\
\Delta \boldsymbol{W}=\left[\begin{array}{llllll}
\Delta w_{1} & \Delta w_{2} & \ldots & \Delta w_{m}
\end{array}\right]^{T}, \quad \Delta \boldsymbol{X}=\left[\begin{array}{lllll}
\Delta x_{1} & \Delta x_{2} & \ldots & \Delta x_{n}
\end{array}\right]^{T}, \quad \Delta \boldsymbol{Y}=\left[\begin{array}{lllll}
\Delta y_{1} & \Delta y_{2} & \ldots & \Delta y_{p}
\end{array}\right]^{T} .
\end{gathered}
$$

The system of Eqs (2.3) can be rewritten in the matrix form

$$
A_{p 0} \cdot \Delta W+B_{p 0} \cdot \Delta X+C_{p 0} \cdot \Delta Y=0 .
$$

Rearranging Eq.(2.4) yields the output position errors

$$
\Delta Y=C_{p 0}^{-1} \cdot\left(-A_{p 0} \cdot \Delta W-B_{p 0} \cdot \Delta X\right)
$$

It can be easily demonstrated that the matrix equation yielding the output velocity will have the form

$$
\dot{Y}=C_{p 0}^{-1} \cdot\left(-B_{p 0} \cdot \dot{X}\right)
$$

\subsection{Velocity errors}

The system of equations needed to determine the velocity errors is obtained by differentiating the system of Eqs (2.1) with respect to time, which gives 


$$
\dot{\boldsymbol{F}}(\boldsymbol{W}, \boldsymbol{X}, \dot{X}, \boldsymbol{Y}, \dot{\boldsymbol{Y}})=\boldsymbol{O}
$$

The velocity error equations can be written as

$$
A_{v 0} \cdot \Delta W+B_{v 0} \cdot \Delta X+B_{v 1} \cdot \Delta \dot{X}+C_{v 0} \cdot \Delta Y+C_{v 1} \cdot \Delta \dot{Y}=0 .
$$

where: $\boldsymbol{A}_{\boldsymbol{v} 0}, \boldsymbol{B}_{\boldsymbol{v} 0}, \boldsymbol{B}_{\boldsymbol{v} 1}, \boldsymbol{C}_{\boldsymbol{v} \boldsymbol{0}}, \boldsymbol{C}_{\boldsymbol{v} 1}$ - denote respective Jacobian matrixes,

$\Delta \dot{\boldsymbol{X}}=\left[\begin{array}{llll}\Delta \dot{x}_{1} & \Delta \dot{x}_{2} & \ldots & \Delta \dot{x}_{n}\end{array}\right]^{T}$ - column matrix of errors in input velocities,

$\Delta \dot{\boldsymbol{Y}}=\left[\begin{array}{llll}\Delta \dot{y}_{1} & \Delta \dot{y}_{2} & \ldots & \Delta \dot{y}_{p}\end{array}\right]^{T}$ - column matrix of errors in output velocities.

Finally, the velocity errors (output kinematic parameters) are derived from the formula

$$
\Delta \dot{\boldsymbol{Y}}=C_{v 1}^{-1} \cdot\left(-A_{v 0} \cdot \Delta W-B_{v 0} \cdot \Delta X-B_{v 1} \cdot \Delta \dot{X}-C_{v 0} \cdot \Delta Y\right) .
$$

Accordingly, the matrix equation used to find the output accelerations can be written in the form

$$
\ddot{Y}=C_{v 1}^{-1} \cdot\left(-B_{v 0} \cdot \dot{X}-B_{v 1} \cdot \ddot{X}-C_{v 0} \cdot \dot{Y}\right) .
$$

\subsection{Acceleration errors}

The system of equations needed to determine the acceleration errors is obtained by differentiating the system of Eq.(2.7) with respect to time, yielding

$$
\ddot{F}(W, X, \dot{X}, \ddot{X}, Y, \dot{Y}, \ddot{Y})=0 .
$$

The matrix equation can be written as

$$
A_{a 0} \cdot \Delta \boldsymbol{W}+\boldsymbol{B}_{a 0} \cdot \Delta \boldsymbol{X}+\boldsymbol{B}_{a 1} \cdot \Delta \dot{X}+B_{a 2} \cdot \Delta \ddot{X}+C_{a 0} \cdot \Delta Y+C_{a 1} \cdot \Delta \dot{Y}+C_{a 2} \cdot \Delta \ddot{Y}=0
$$

where: $\boldsymbol{A}_{\boldsymbol{a} \boldsymbol{0}}, \boldsymbol{B}_{\boldsymbol{a} \boldsymbol{0}}, \boldsymbol{B}_{\boldsymbol{a} 1}, \boldsymbol{B}_{\boldsymbol{a} 2}, \boldsymbol{C}_{\boldsymbol{a} \boldsymbol{0}}, \boldsymbol{C}_{\boldsymbol{a} \boldsymbol{l}}, \boldsymbol{C}_{\boldsymbol{a} 2}$ - respective Jacobian matrixes,

$\Delta \ddot{\boldsymbol{X}}=\left[\begin{array}{llll}\Delta \ddot{x}_{1} & \Delta \ddot{x}_{2} & \ldots & \Delta \ddot{x}_{n}\end{array}\right]^{T}$ - column matrix of errors in input accelerations,

$\Delta \ddot{\boldsymbol{Y}}=\left[\begin{array}{llll}\Delta \ddot{y}_{1} & \Delta \ddot{y}_{2} & \ldots & \Delta \ddot{y}_{n}\end{array}\right]^{T}$ - column matrix of errors in output accelerations.

Rearranging Eq.(2.12), we get the errors in output accelerations

$$
\Delta \ddot{\boldsymbol{Y}}=\boldsymbol{C}_{a 2}^{-1}\left(-\boldsymbol{A}_{a 0} \cdot \Delta \boldsymbol{W}-\boldsymbol{B}_{a 0} \cdot \Delta \boldsymbol{X}-\boldsymbol{B}_{a 1} \cdot \Delta \dot{\boldsymbol{X}}-\boldsymbol{B}_{a 2} \cdot \Delta \ddot{\boldsymbol{X}}-\boldsymbol{C}_{a 0} \cdot \Delta \boldsymbol{Y}-\boldsymbol{C}_{a 1} \cdot \Delta \dot{\boldsymbol{Y}}\right) .
$$

Matrix equations to find the jerk errors (output quantities) can be written as

$$
\dddot{Y}=C_{a 2}^{-1}\left(-B_{a 0} \cdot \dot{X}-B_{a 1} \cdot \ddot{X}-B_{a 2} \cdot \dddot{X}-C_{a 0} \cdot \dot{Y}-C_{a 1} \cdot \ddot{Y}\right) .
$$




\subsection{Jerk errors}

The system of equations needed to determine the jerk errors is obtained by differentiating the system of Eqs (2.11) with respect to time, yielding

$$
\dddot{F}(W, X, \dot{X}, \ddot{X}, \dddot{X}, Y, \dot{Y}, \ddot{Y}, \dddot{Y})=0
$$

Jerk error equations can be written as

$$
\begin{aligned}
& A_{u 0} \cdot \Delta \boldsymbol{W}+\boldsymbol{B}_{u 0} \cdot \Delta \boldsymbol{X}+\boldsymbol{B}_{u 1} \cdot \Delta \dot{\boldsymbol{X}}+\boldsymbol{B}_{u 2} \cdot \Delta \ddot{\boldsymbol{X}}+\boldsymbol{B}_{u 3} \cdot \Delta \dddot{\boldsymbol{X}}+ \\
& +\boldsymbol{C}_{u 0} \cdot \Delta \boldsymbol{Y}+\boldsymbol{C}_{u 1} \cdot \Delta \dot{\boldsymbol{Y}}+\boldsymbol{C}_{u 2} \cdot \Delta \ddot{\boldsymbol{Y}}+\boldsymbol{C}_{u 3} \cdot \Delta \dddot{\boldsymbol{Y}}=\mathbf{0}
\end{aligned}
$$

where: $\boldsymbol{A}_{\boldsymbol{u} \boldsymbol{0}}, \boldsymbol{B}_{\boldsymbol{u} \boldsymbol{0}}, \boldsymbol{B}_{\boldsymbol{u} \boldsymbol{1}}, \boldsymbol{B}_{\boldsymbol{u} \boldsymbol{2}}, \boldsymbol{B}_{\boldsymbol{u} 3}, \boldsymbol{C}_{\boldsymbol{u} \boldsymbol{0}}, \boldsymbol{C}_{\boldsymbol{u} \boldsymbol{1}}, \boldsymbol{C}_{\boldsymbol{u} \mathbf{2}}, \boldsymbol{C}_{\boldsymbol{u} \mathbf{3}}$ - respective Jacobian matrixes,

$\Delta \dddot{\boldsymbol{X}}=\left[\begin{array}{llll}\Delta \dddot{x}_{1} & \Delta \dddot{x}_{2} & \ldots & \Delta \dddot{x}_{n}\end{array}\right]^{T}$ - column matrix of errors in input jerks,

$\Delta \dddot{\boldsymbol{Y}}=\left[\begin{array}{llll}\Delta \dddot{y}_{1} & \Delta \dddot{y}_{2} & \ldots & \Delta \dddot{y}_{p}\end{array}\right]^{T}$ - column matrix of errors in output jerks.

Rearranging Eq.(2.16) yields errors in output jerks

$$
\begin{aligned}
& \Delta \dddot{Y}=C_{u 3}^{-1} \cdot\left(-A_{u 0} \cdot \Delta W-B_{u 0} \cdot \Delta X-B_{u 1} \cdot \Delta \dot{X}-B_{u 2} \cdot \Delta \ddot{X}+\right. \\
& \left.-B_{u 3} \cdot \Delta \dddot{X}-C_{u 0} \cdot \Delta Y-C_{u 1} \cdot \Delta \dot{Y}-C_{u 2} \cdot \Delta \ddot{Y}\right)
\end{aligned}
$$

It is readily apparent that

$$
\boldsymbol{B}_{p 0}=\boldsymbol{B}_{v 1}=\boldsymbol{B}_{a 2}=\boldsymbol{B}_{u 3} \quad \text { and } \quad \boldsymbol{C}_{p 0}=\boldsymbol{C}_{v 1}=\boldsymbol{C}_{a 2}=\boldsymbol{C}_{u 3} .
$$

\section{Determining the errors in kinematic parameters using the kinematic software library}

When investigating mechanisms incorporating a number of kinematic units, the recommended method is that using the kinematic software library (Młynarski, 1994), expanded accordingly to handle the errors of kinematic parameters. Figure 1 shows a kinematic diagram of an aerial device. The operator's comfort in this machine is affected by the error of the basket floor's angular position (2.8) and acceleration of the basket floor. The extreme deviations of acceleration are of particular interest. Obviously, the answer to some of these problems will be available after creating and testing the dynamic models taking into account the behaviour of the machine as a whole (with all implements) as well as ground flexibility. Nevertheless, the relationships provided in this work can be most useful in further calculations, for example in calculating the static positioning errors. The kinematic system shown in Fig. 1 comprises four class II kinematic units numbered in accordance with the sequence applied in the calculation procedure, though in the case considered here the units 2 and 3 can be handled in any order. The drive system incorporates two actuators $(2,6)$. Errors in kinematic parameters of the link 8 (basket) can be determined by performing calculations for individual kinematic units whilst the output kinematic parameters and involved errors in one kinematic unit can become the inputs to the kinematic units to be handled next. Errors in kinematic parameters of particular units are determined using the same procedure as in the general case. The kinematic 
software library can handle other methods of link dimensioning than that shown in Fig.1. Solving the task illustrated in Fig.1 requires two types of class II kinematic units, as demonstrated below.

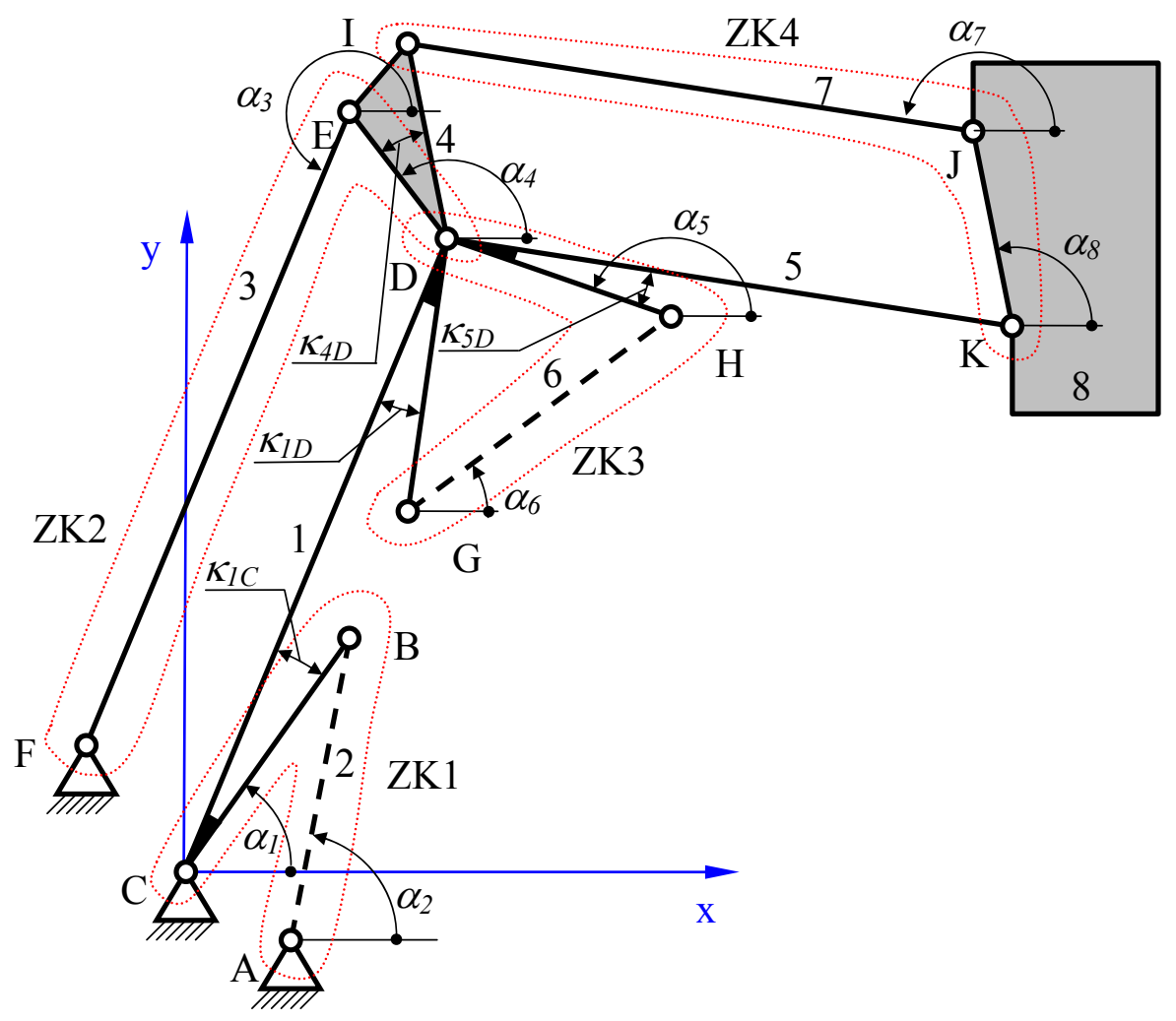

Fig.1. Schematic diagram of an aerial device.

Figure 2 shows a class II kinematic unit with indicated attachment points D and E, link and point designations do not coincide with those in Fig.1. Equations of attachment points' positions can be incorporated into the system of Eqs (2.1) however, it seems an unjustified procedure because the number of equations will increase. Alternatively, the kinematic unit can be handled separately and kinematic parameters of attachment points and the involved errors should be determined in the next step.

Input data include

$$
\begin{aligned}
& \boldsymbol{W}=\left[\begin{array}{lll}
l_{A B} & l_{B C}
\end{array}\right]^{T}, \quad \Delta \boldsymbol{W}=\left[\begin{array}{lll}
\Delta l_{A B} & \Delta l_{B C}
\end{array}\right]^{T}, \quad \boldsymbol{X}=\left[\begin{array}{llll}
x_{A} & y_{A} & x_{C} & y_{C}
\end{array}\right]^{T}, \\
& \Delta \boldsymbol{X}=\left[\begin{array}{lllll}
\Delta x_{A} & \Delta y_{A} & \Delta x_{C} & \Delta y_{C}
\end{array}\right]^{T}, \quad \dot{\boldsymbol{X}}=\left[\begin{array}{lllll}
\dot{x}_{A} & \dot{y}_{A} & \dot{x}_{C} & \dot{y}_{C}
\end{array}\right]^{T}, \\
& \Delta \dot{\boldsymbol{X}}=\left[\begin{array}{llllll}
\Delta \dot{x}_{A} & \Delta \dot{y}_{A} & \Delta \dot{x}_{C} & \Delta \dot{y}_{C}
\end{array}\right]^{T}, \quad \ddot{\boldsymbol{X}}=\left[\begin{array}{lllll}
\ddot{x}_{A} & \ddot{y}_{A} & \ddot{x}_{C} & \ddot{y}_{C}
\end{array}\right]^{T}, \\
& \Delta \ddot{\boldsymbol{X}}=\left[\begin{array}{llllll}
\Delta \ddot{x}_{A} & \Delta \ddot{y}_{A} & \Delta \ddot{x}_{C} & \Delta \ddot{y}_{C}
\end{array}\right]^{T}, \quad \dddot{\boldsymbol{X}}=\left[\begin{array}{lllll}
\dddot{x}_{A} & \dddot{y}_{A} & \dddot{x}_{C} & \dddot{y}_{C}
\end{array}\right]^{T}, \\
& \Delta \dddot{\boldsymbol{X}}=\left[\begin{array}{lllll}
\Delta \dddot{x}_{A} & \Delta \dddot{y}_{A} & \Delta \dddot{x}_{C} & \Delta \dddot{y}_{C}
\end{array}\right]^{T} .
\end{aligned}
$$


Output quantities to be determined are

$$
\begin{array}{cl}
\boldsymbol{Y}=\left[\begin{array}{ll}
\alpha_{1} & \alpha_{2}
\end{array}\right]^{T}, \quad \Delta \boldsymbol{Y}=\left[\begin{array}{ll}
\Delta \alpha_{1} & \Delta \alpha_{2}
\end{array}\right]^{T}, \quad \dot{\boldsymbol{Y}}=\left[\begin{array}{ll}
\dot{\alpha}_{1} & \dot{\alpha}_{2}
\end{array}\right]^{T}, \quad \Delta \dot{\boldsymbol{Y}}=\left[\begin{array}{ll}
\Delta \dot{\alpha}_{1} & \Delta \dot{\alpha}_{2}
\end{array}\right]^{T}, \\
\ddot{\boldsymbol{Y}}=\left[\begin{array}{ll}
\ddot{\alpha}_{1} & \ddot{\alpha}_{2}
\end{array}\right]^{T,}, \quad \Delta \ddot{\boldsymbol{Y}}=\left[\begin{array}{lll}
\Delta \ddot{\alpha}_{1} & \Delta \ddot{\alpha}_{2}
\end{array}\right]^{T,}, \quad \dddot{\boldsymbol{Y}}^{T}=\left[\begin{array}{ll}
\dddot{\alpha}_{1} & \dddot{\alpha}_{2}
\end{array}\right]^{T,}, \quad \Delta \dddot{\boldsymbol{Y}}=\left[\begin{array}{ll}
\Delta \dddot{\alpha}_{1} & \Delta \dddot{\alpha}_{2}
\end{array}\right]^{T} .
\end{array}
$$

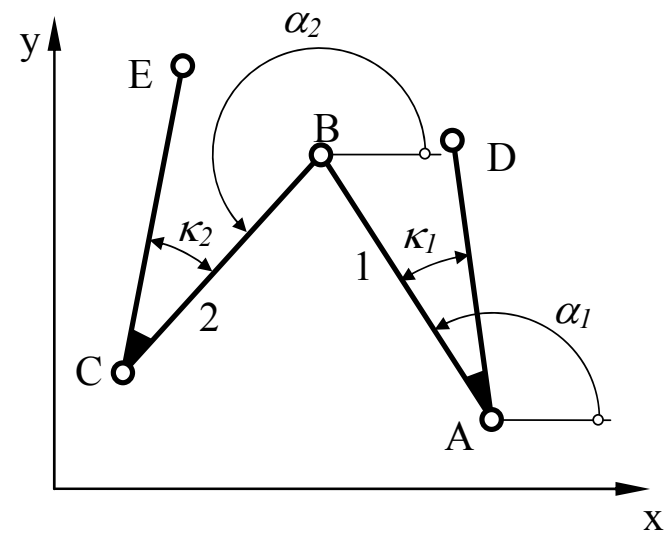

Fig.2. Class II kinematic unit.

Equations governing the position of the kinematic unit become

$$
\left\{\begin{array}{l}
f_{1}=x_{A}-x_{C}+l_{A B} \cos \left(\alpha_{1}\right)+l_{B C} \cos \left(\alpha_{2}\right)=0 \\
f_{2}=y_{A}-y_{C}+l_{A B} \sin \left(\alpha_{1}\right)+l_{B C} \sin \left(\alpha_{2}\right)=0 .
\end{array}\right.
$$

Solving the system of Eqs (3.1) yields $\mathbf{Y}$ and differentiating the system of Eqs (3.1) with respect to time, we get

$$
\left\{\begin{array}{l}
\dot{f}_{1}=\dot{x}_{A}-\dot{x}_{C}-l_{A B} \dot{\alpha}_{1} \sin \left(\alpha_{1}\right)-l_{B C} \dot{\alpha}_{2} \sin \left(\alpha_{2}\right)=0 \\
\dot{f}_{2}=\dot{y}_{A}-\dot{y}_{C}+l_{A B} \dot{\alpha}_{1} \cos \left(\alpha_{1}\right)+l_{B C} \dot{\alpha}_{2} \cos \left(\alpha_{2}\right)=0
\end{array}\right.
$$

Differentiating the system of Eqs (3.2) with respect to time gives

$$
\left\{\begin{array}{l}
\ddot{f}_{1}=\ddot{x}_{A}-\ddot{x}_{C}-l_{A B}\left(\ddot{\alpha}_{1} \sin \left(\alpha_{1}\right)+\dot{\alpha}_{1}^{2} \cos \left(\alpha_{1}\right)\right)-l_{B C}\left(\ddot{\alpha}_{2} \sin \left(\alpha_{2}\right)+\dot{\alpha}_{2}^{2} \cos \left(\alpha_{2}\right)\right)=0, \\
\ddot{f}_{2}=\ddot{y}_{A}-\ddot{y}_{C}+l_{A B}\left(\ddot{\alpha}_{1} \cos \left(\alpha_{1}\right)-\dot{\alpha}_{1}^{2} \sin \left(\alpha_{1}\right)\right)+l_{B C}\left(\ddot{\alpha}_{2} \cos \left(\alpha_{2}\right)-\dot{\alpha}_{2}^{2} \sin \left(\alpha_{2}\right)\right)=0 .
\end{array}\right.
$$

Differentiating the system of Eqs (3.4) with respect to time we get 


$$
\left\{\begin{array}{l}
\dddot{f}_{1}=\dddot{x}_{A}-\dddot{x}_{C}-l_{A B}\left(\dddot{\alpha}_{1} \sin \left(\alpha_{1}\right)+3 \dot{\alpha}_{1} \ddot{\alpha}_{1} \cos \left(\alpha_{1}\right)-\dot{\alpha}_{1}^{3} \sin \left(\alpha_{1}\right)\right)+ \\
-l_{B C}\left(\dddot{\alpha}_{2} \sin \left(\alpha_{2}\right)+3 \dot{\alpha}_{2} \ddot{\alpha}_{2} \cos \left(\alpha_{2}\right)-\dot{\alpha}_{2}^{3} \sin \left(\alpha_{2}\right)\right)=0, \\
\dddot{f}_{2}=\dddot{y}_{A}-\dddot{y}_{C}+l_{A B}\left(\dddot{\alpha}_{1} \cos \left(\alpha_{1}\right)-3 \dot{\alpha}_{1} \ddot{\alpha}_{1} \sin \left(\alpha_{1}\right)-\dot{\alpha}_{1}^{3} \cos \left(\alpha_{1}\right)\right)+ \\
+l_{B C}\left(\dddot{\alpha}_{2} \cos \left(\alpha_{2}\right)-3 \dot{\alpha}_{2} \ddot{\alpha}_{2} \sin \left(\alpha_{1}\right)-\dot{\alpha}_{2}^{3} \cos \left(\alpha_{2}\right)\right)=0 .
\end{array}\right.
$$

To find the position errors (output parameters), the respective matrixes need to be determined

$$
\boldsymbol{A}_{\boldsymbol{p} \boldsymbol{0}}=\left[\begin{array}{cc}
\cos \left(\alpha_{1}\right) & \cos \left(\alpha_{2}\right) \\
\sin \left(\alpha_{1}\right) & \sin \left(\alpha_{2}\right)
\end{array}\right], \quad \boldsymbol{B}_{\boldsymbol{p} \boldsymbol{0}}=\left[\begin{array}{cccc}
1 & 0 & -1 & 0 \\
0 & 1 & 0 & -1
\end{array}\right], \quad \boldsymbol{C}_{\boldsymbol{p} \boldsymbol{0}}=\left[\begin{array}{cc}
-l_{A B} \sin \left(\alpha_{1}\right) & -l_{B C} \sin \left(\alpha_{2}\right) \\
l_{A B} \cos \left(\alpha_{1}\right) & l_{B C} \cos \left(\alpha_{2}\right)
\end{array}\right] .
$$

Position errors $\Delta \boldsymbol{Y}$ are obtained from Eq.(2.5). The procedure to determine the position errors of attachment points is shown using the example of the point $\mathrm{D}$ whose co-ordinates can be derived from the equations below

$$
\left\{\begin{array}{l}
x_{D}=x_{A}+l_{A D} \cos \left(\alpha_{1}-\kappa_{1}\right) \\
y_{D}=y_{A}+l_{A D} \sin \left(\alpha_{1}-\kappa_{1}\right)
\end{array}\right.
$$

Recalling the linear theory of errors, we get $\Delta x_{D}, \Delta y_{D}$. It is worthwhile to mention that all quantities on the right-hand side of the equation are known beforehand and so are the involved errors.

To find the errors in output velocities, the respective matrixes are derived

$$
\begin{aligned}
& \boldsymbol{A}_{\boldsymbol{v} \boldsymbol{0}}=\left[\begin{array}{cc}
-\dot{\alpha}_{1} \sin \left(\alpha_{1}\right) & -\dot{\alpha}_{2} \sin \left(\alpha_{2}\right) \\
\dot{\alpha}_{1} \cos \left(\alpha_{1}\right) & \dot{\alpha}_{2} \cos \left(\alpha_{2}\right)
\end{array}\right], \quad \boldsymbol{C}_{\boldsymbol{v} \boldsymbol{0}}=\left[\begin{array}{cc}
-l_{A B} \dot{\alpha}_{1} \cos \left(\alpha_{1}\right) & -l_{B C} \dot{\alpha}_{2} \cos \left(\alpha_{2}\right) \\
l_{A B} \dot{\alpha}_{1} \sin \left(\alpha_{1}\right) & l_{B C} \dot{\alpha}_{2} \sin \left(\alpha_{2}\right)
\end{array}\right], \\
& \boldsymbol{B}_{\boldsymbol{v} \boldsymbol{0}}=\boldsymbol{0}, \quad \boldsymbol{B}_{\boldsymbol{v} \boldsymbol{1}}=\boldsymbol{B}_{\boldsymbol{p} \boldsymbol{0}}, \quad \boldsymbol{C}_{\boldsymbol{v} \boldsymbol{1}}=\boldsymbol{C}_{\boldsymbol{p} \boldsymbol{0}} .
\end{aligned}
$$

Velocity errors $\Delta \dot{\boldsymbol{Y}}$ are derived from formula (2.9). In the case of point D, Eqs (3.5) must be differentiated with respect to time and $\Delta \dot{x}_{D}, \Delta \dot{y}_{D}$ can be obtained.

To determine the errors in output accelerations, the relevant matrixes are written as follows

$$
\boldsymbol{A}_{a \boldsymbol{0}}, \boldsymbol{B}_{a \boldsymbol{0}}, \boldsymbol{B}_{a 1}, \boldsymbol{B}_{a 2}=\boldsymbol{B}_{\boldsymbol{p} \boldsymbol{0}}, \boldsymbol{C}_{a \boldsymbol{0}}, \boldsymbol{C}_{a 1}, \boldsymbol{C}_{a 2}=\boldsymbol{C}_{\boldsymbol{p} \boldsymbol{0}}
$$

Acceleration errors $\Delta \ddot{\boldsymbol{Y}}$ are derived from formula (2.13). When handling point D, Eqs (3.5) should be differentiated twice with respect to time and $\Delta \ddot{x}_{D}, \Delta \ddot{y}_{D}$ can be obtained accordingly.

Figure 3 illustrates a class II kinematic unit with an internal drive (actuator). 


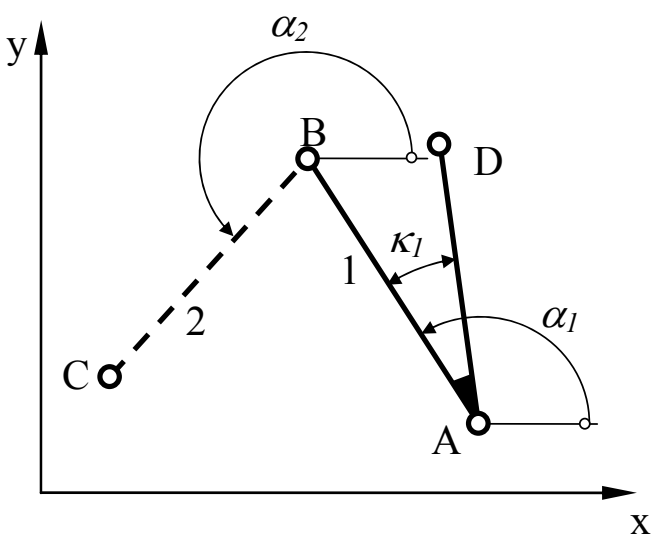

Fig.3. A class II kinematic unit with an actuator.

Input data include

$$
\begin{aligned}
& \boldsymbol{W}=l_{A B}, \quad \Delta \boldsymbol{W}=\Delta l_{A B}, \quad \boldsymbol{X}=\left[\begin{array}{lllll}
x_{A} & y_{A} & x_{B} & y_{B} & s_{B C}
\end{array}\right]^{T}, \\
& \Delta \boldsymbol{X}=\left[\begin{array}{llllll}
\Delta x_{A} & \Delta y_{A} & \Delta x_{B} & \Delta y_{B} & \Delta s_{B C}
\end{array}\right]^{T}, \quad \dot{\boldsymbol{X}}, \Delta \dot{\boldsymbol{X}}, \ddot{\boldsymbol{X}}, \Delta \ddot{\boldsymbol{X}}, \dddot{\boldsymbol{X}}, \Delta \dddot{\boldsymbol{X}} .
\end{aligned}
$$

The quantities to be determined are

$$
\boldsymbol{Y}=\left[\begin{array}{ll}
\alpha_{1} & \alpha_{2}
\end{array}\right]^{T}, \quad \Delta \boldsymbol{Y}=\left[\begin{array}{ll}
\Delta \alpha_{1} & \Delta \alpha_{2}
\end{array}\right]^{T}, \quad \dot{\boldsymbol{Y}}, \Delta \dot{\boldsymbol{Y}}, \ddot{\boldsymbol{Y}}, \Delta \ddot{\boldsymbol{Y}}, \ddot{\boldsymbol{Y}}, \Delta \dddot{\boldsymbol{Y}} .
$$

The equations of the kinematic links' positions can be written as

$$
\left\{\begin{array}{l}
f_{1}=x_{A}-x_{C}+l_{A B} \cos \left(\alpha_{1}\right)+s_{B C} \cos \left(\alpha_{2}\right)=0 \\
f_{2}=y_{A}-y_{C}+l_{A B} \sin \left(\alpha_{1}\right)+s_{B C} \sin \left(\alpha_{2}\right)=0
\end{array}\right.
$$

The procedure to be adopted in further calculations has been outlined in previous sections.

\section{Conclusion}

In many cases the actual values of input quantities are unknown and only their deviations are available. The upper and lower limit of admissible errors in output kinematic parameters can be determined using the procedure outlined in Kotulski and Szczepiński (2004), however it is required that the impact factors expressing how the given error type affects the final result should be first established. It is achieved by multiplying the relevant matrixes in Eqs (2.5), (2.9), (2.13), (2.17).

Recalling Eqs (2.18), it appears that to determine all errors in output kinematic parameters it suffices that the inverse matrix to only one matrix $\boldsymbol{C}_{\boldsymbol{p} \theta}$ should be computed, which requires a numerical procedure. In the case of matrices with small dimensions this problem can be solved using symbols (for example for class II mechanisms).

In the case of cam and lift mechanisms, the kinematic analysis of mechanical systems typically involves the jerk analysis as well, whilst theoretical works addressing those aspects are rather scarce. Hence, 
the study of errors in kinematic parameters also includes the jerk analysis. When handling the regions in the neighbourhood of undesired singular positions, it is recommended to replace the linear error analysis with a non-linear theory, to account for the effects that particular errors have on other error types. In certain cases the assumption that links are non- deformed has to be abandoned (as in the case of a mechanism in the knuckle joint press).

The choice of the link dimensioning method can affect the final results, too. When formulating the equations of constraints, the minimal path principle should be adopted. Determining the errors in kinematic parameters of driving links may prove to be a time-consuming task. Errors in input parameters can be determined by theoretical analyses, from testing of virtual models and through measurements of real-life objects.

\section{Nomenclature}

$$
\begin{aligned}
& \boldsymbol{A}_{a \boldsymbol{0}}=\left[\frac{\partial \ddot{f}_{l}}{\partial w_{i}}\right]_{l, i}, \boldsymbol{B}_{a \boldsymbol{0}}=\left[\frac{\partial \ddot{f}_{l}}{\partial x_{j}}\right]_{l, j}, \\
& \boldsymbol{B}_{a 1}=\left[\frac{\partial \ddot{f}_{l}}{\partial \dot{x}_{j}}\right]_{l, j}, \boldsymbol{B}_{a 2}=\left[\frac{\partial \ddot{f}_{l}}{\partial \ddot{x}_{j}}\right]_{l, j}, \\
& \boldsymbol{C}_{a \boldsymbol{\theta}}=\left[\frac{\partial \ddot{f}_{l}}{\partial y_{k}}\right]_{l, k}, \boldsymbol{C}_{a l}=\left[\frac{\partial \ddot{f}_{l}}{\partial \dot{y}_{k}}\right]_{l, k}, \\
& \boldsymbol{C}_{a 2}=\left[\frac{\partial \ddot{f}_{l}}{\partial \ddot{y}_{k}}\right]_{l, k} \\
& \boldsymbol{A}_{\boldsymbol{p} \boldsymbol{0}}=\left[\frac{\partial f_{l}}{\partial w_{i}}\right]_{l, i}, \boldsymbol{B}_{\boldsymbol{p} \boldsymbol{0}}=\left[\frac{\partial f_{l}}{\partial x_{j}}\right]_{l, j}, \\
& \boldsymbol{C}_{\boldsymbol{p} \boldsymbol{0}}=\left[\frac{\partial f_{l}}{\partial y_{k}}\right]_{l, k}, i=1 . . m, \quad-\text { Jacobian matrices in the matrix equation of position errors (2.4) } \\
& j=1 . . n, k=1 . . p, l=1 . . p \\
& \boldsymbol{A}_{u \boldsymbol{0}}=\left[\frac{\partial \dddot{f}_{l}}{\partial w_{i}}\right]_{l, i}, \boldsymbol{B}_{u \boldsymbol{0}}=\left[\frac{\partial \dddot{f}_{l}}{\partial x_{j}}\right]_{l, j}, \\
& \boldsymbol{B}_{u l}=\left[\frac{\partial \ddot{f}_{l}}{\partial \dot{x}_{j}}\right]_{l, j}, \boldsymbol{B}_{u 2}=\left[\frac{\partial \dddot{f}_{l}}{\partial \ddot{x}_{j}}\right]_{l, j}, \\
& \boldsymbol{B}_{u 3}=\left[\frac{\partial \dddot{f}_{l}}{\partial \dddot{x}_{j}}\right]_{l, j}, \boldsymbol{C}_{u \boldsymbol{0}}=\left[\frac{\partial \dddot{f}_{l}}{\partial y_{k}}\right]_{l, k},- \text { Jacobian Matrices in the matrix equation of jerk errors (2.16) } \\
& \boldsymbol{C}_{u 1}=\left[\frac{\partial \ddot{f}_{l}}{\partial \dot{y}_{k}}\right]_{l, k}, \boldsymbol{C}_{u 2}=\left[\frac{\partial \dddot{f}_{l}}{\partial \ddot{y}_{k}}\right]_{l, k}, \\
& \boldsymbol{C}_{u 3}=\left[\frac{\partial \dddot{f}_{l}}{\partial \ddot{y}_{k}}\right]_{l, k} \\
& \boldsymbol{A}_{v \boldsymbol{0}}=\left[\frac{\partial \dot{f}_{l}}{\partial w_{i}}\right]_{l, i}, \boldsymbol{B}_{v \boldsymbol{0}}=\left[\frac{\partial \dot{f}_{l}}{\partial x_{j}}\right]_{l, j} \\
& \boldsymbol{B}_{v l}=\left[\frac{\partial \dot{f}_{l}}{\partial \dot{x}_{j}}\right]_{l, j}, \boldsymbol{C}_{v \boldsymbol{0}}=\left[\frac{\partial \dot{f}_{l}}{\partial y_{k}}\right]_{l, k},- \text { Jacobian matrices in the matrix equation of velocity errors (2.8) } \\
& \boldsymbol{C}_{v l}=\left[\frac{\partial \dot{f}_{l}}{\partial \dot{y}_{k}}\right]_{l, k} \\
& \text { - Jacobian matrices in the matrix equation of acceleration errors (2.12) }
\end{aligned}
$$


$\boldsymbol{F}, \dot{\boldsymbol{F}}, \ddot{\boldsymbol{F}}, \dddot{\boldsymbol{F}} \quad$ - column matrix of geometric constraint equations and its time derivatives

$t$ - time

$\boldsymbol{W}$ - column matrix of (time -independent) basic geometric dimensions

$\boldsymbol{X}, \dot{X}, \ddot{X}, \dddot{X} \quad$ - column matrices of (time-dependent) input kinematic parameters

$\boldsymbol{Y}, \dot{\boldsymbol{Y}}, \ddot{\boldsymbol{Y}}, \dddot{\boldsymbol{Y}}$ - column matrices of (time-dependent) output kinematic parameters

$\Delta \mathbf{W}$ - column matrix of errors in basic geometric dimensions

$\Delta \boldsymbol{X}, \Delta \dot{\boldsymbol{X}}, \Delta \ddot{\boldsymbol{X}}, \Delta \dddot{\boldsymbol{X}}-$ column matrices of errors in respective input parameters $\boldsymbol{X}, \dot{\boldsymbol{X}}, \ddot{X}, \dddot{X}$

$\Delta \boldsymbol{Y}, \Delta \dot{\boldsymbol{Y}}, \Delta \ddot{\boldsymbol{Y}}, \Delta \dddot{\boldsymbol{Y}}-$ column matrices of errors in respective output parameters $\boldsymbol{Y}, \dot{\boldsymbol{Y}}, \ddot{\boldsymbol{Y}}, \dddot{\boldsymbol{Y}}$

\section{References}

Fenton R.G., Cleghorn W.L. and Jing-Fan Fu (1989): Allocation of Dimensional Tolerances for Multiple Loop Planar Mechanisms. - Journal of Mechanisms, Transmissions and Automation in Design, XII 1989, vol.111.

Gronowicz A. (2003): Fundamentals of Analysis of Kinematic Systems. - Wroclaw Polytechnics Publishers, Wrocław (In Polish).

Kotulski Z. and Szczepiński W. (2004): Calculus of Errors for Engineers. - WNT Publishers, Warsaw (In Polish).

Mavroidis C., Flanz J., Dubowsky S., Drouet P. and Goiltein M. (1998): High Performance Medical Robot Requirements and Accuracy Analysis. - Robotics and Computer Integrated Manufacturing, vol.14, No.5-6, pp.329338.

Młynarski T. (1994): Generalised Analytical Method of Kinematic Analysis of Plane Mechanisms. - Cracow University of Technology, Monograph 165, Cracow (In Polish).

Received: June 2, 2014

Revised: October 1, 2014 